\title{
THE UNIFORM CLT FOR MARTINGALE DIFFERENCE ARRAYS UNDER THE UNIFORMLY INTEGRABLE ENTROPY
}

\author{
Jongsig Bae, Doobae Jun, and Shlomo Levental
}

\begin{abstract}
In this paper we consider the uniform central limit theorem for a martingale-difference array of a function-indexed stochastic process under the uniformly integrable entropy condition. We prove a maximal inequality for martingale-difference arrays of process indexed by a class of measurable functions by a method as Ziegler [19] did for triangular arrays of row wise independent process. The main tools are the Freedman inequality for the martingale-difference and a sub-Gaussian inequality based on the restricted chaining. The results of present paper generalizes those of Ziegler [19] and other results of independent problems. The results also generalizes those of Bae and Choi [3] to martingale-difference array of a function-indexed stochastic process. Finally, an application to classes of functions changing with $n$ is given.
\end{abstract}

\section{Introduction}

In the present paper we deal with the uniform central limit theorem for a specific function-indexed process based on a martingale-difference array under the assumption of uniformly integrable entropy.

Several extensions from the classical Lindeberg-Feller models are possible. One direction is to consider the process version of the central limit theorem pioneered by Donsker [6]. The index set is evolved from the real line to a function class which can be considered as a general metric space. The so called function-indexed process has turned out to be a natural extension keeping in mind in applications in random fields. See, for example, Adler [1] for general Gaussian random fields, Bass for set-indexed processes, and Dudley [8] for function-indexed processes.

The other direction is to remove independence assumption by considering problems such as martingales, stationary Markov chains and various types of mixing sequences. Numerous development on this points have been made since

Received August 11, 2008; Revised February 4, 2009.

2000 Mathematics Subject Classification. Primary 60F17; Secondary 60F05.

Key words and phrases. uniform CLT, martingale difference array, uniformly integrable entropy, restricted chaining, sequential empirical process. 
1970's. See, for example, Gordin and Lifsic [12], Bae and Levental [4], and Doukhan et al. [7].

When one deals with the uniform CLT for a function-indexed process, bracketing method and random entropy method are commonly used in the literature. Under one of these assumptions, most widely used tool is the chaining argument. The last argument is originated, to the best of our knowledge, from Kolmogorov. See for example Kolmogorov's lemma in probability texts. For the use of chaining argument for the set-indexed process, see Bass [5]. A more delicate chaining argument with stratifications is employed in Ossiander [14]. Most recent texts on these topics are Dudley [9] and van de Geer [17]. The present paper is motivated by Bae [2] and Ziegler [19]. The first paper deals with the uniform CLT for a sequence of stationary martingale difference under the assumption of bracketing entropy and the second one deals with functional CLTs for row wise independent triangular arrays of function-indexed processes under the uniformly integrable entropy condition.

In the present paper we do not attempt to use the chaining argument directly in developing the uniform CLT, but we prove a maximal inequality that will give the uniform CLT by an approach as Ziegler [19] employed for triangular arrays of row wise independent processes. We believe that our approach to the martingale problem is substantially different from those of Ziegler's independent problem. For instance, the symmetrization argument is not work for a dependent process.

Consider an array of sub- $\sigma$-fields $\left\{\mathcal{E}_{n j}: 0 \leq j \leq j(n), n \in \mathbb{N}\right\}$ on a given probability space $(\Omega, \mathcal{E}, P)$ satisfying $\mathcal{E}_{n 0} \subseteq \mathcal{E}_{n 1} \subseteq \cdots \subseteq \mathcal{E}_{n j(n)}$ for $n \in \mathbb{N}$. Given a collection $\mathcal{F}$ of real-valued functions defined on a measurable space $(\mathbf{X}, \mathcal{X})$, we consider an array $\left\{V_{n j}(f): j \leq j(n), n \in \mathbb{N}, f \in \mathcal{F}\right\}$ of martingale-difference of $\mathcal{L}_{2}$-process indexed by $\mathcal{F}$ with respect to the $\sigma$-fields $\left\{\mathcal{E}_{n j}: 0 \leq j \leq n, n \in \mathbb{N}\right\}$. By that we mean that, for each $f \in \mathcal{F},\left\{V_{n j}(f): j \leq j(n), n \in \mathbb{N}\right\}$ is an array of random variables satisfying $E\left(V_{n j}(f) \mid \mathcal{E}_{n, j-1}\right)=0$ and $V_{n j}(f)$ is $\mathcal{E}_{n j^{-}}$ measurable.

We simply denote $E_{n, j-1} f$ to mean $E\left(f \mid \mathcal{E}_{n, j-1}\right)$, the conditional expectation of the random element $f$ given the $\sigma$-field $\mathcal{E}_{n, j-1}$. Define a conditional variance process $v_{n j}(f):=E_{n, j-1}\left(V_{n j}(f)\right)^{2}$ for $f \in \mathcal{F}$. Notice that $v_{n j}(f)$ is an $\mathcal{E}_{n, j-1^{-}}$ measurable random variable.

Given a class $\mathcal{F}$ of measurable functions defined on a measurable space $(\mathbf{X}, \mathcal{X})$, the covering number $N(\epsilon, \mathcal{F},\|\cdot\|)$, simply denote $N(\epsilon)$ when there is no risk of ambiguity, is the minimum number of balls $\{g:\|g-h\|<\epsilon\}$ of radius $\epsilon$ needed to cover $\mathcal{F}$. Let $F$ be an envelope of $\mathcal{F}$. That is, $F$ is a measurable function from $\mathbf{X}$ to $[0, \infty)$ such that $\sup _{f \in \mathcal{F}}|f(x)| \leq F(x)$ for all $x \in \mathbf{X}$. Let $M(\mathbf{X}, F)$ be the set of all measures $\gamma$ on $(\mathbf{X}, \mathcal{X})$ with $\gamma\left(F^{2}\right):=\int_{\mathbf{X}} F^{2} d \gamma<\infty$.

Given random measures $\mu_{n}$ on $(\mathbf{X}, \mathcal{X})$, we define

$$
d_{\mu_{n}}^{(2)}(f, g):=\left[\mu_{n}(f-g)^{2}\right]^{1 / 2} .
$$


Say that $\mathcal{F}$ has uniformly integrable entropy with respect to $\mathcal{L}_{2}$-norm if

$$
\int_{0}^{\infty} \sup _{\gamma \in M(\mathbf{X}, F)}\left[\ln N\left(\epsilon\left[\gamma\left(F^{2}\right)\right]^{1 / 2}, \mathcal{F}, d_{\gamma}^{(2)}\right)\right]^{1 / 2} d \epsilon<\infty,
$$

where $d_{\gamma}^{(2)}(f, g):=\left[\int_{\mathbf{X}}(f-g)^{2} d \gamma\right]^{1 / 2}$. When the class $\mathcal{F}$ has uniformly integrable entropy, $\left(\mathcal{F}, d_{\gamma}^{(2)}\right)$ is totally bounded for any measure $\gamma$. Many important classes of functions, such as VC graph classes, have uniformly integrable entropy. See Section 2.6 of Van der Vaart and Wellner [18].

The one dimensional CLT for a martingale-difference array deals with convergence in distribution to a normal random variable of row sums of the martingale-difference array. See Theorem 1 in Chapter 8 of Pollard [15] among others.

The goal of this paper is to establish a uniform CLT for an array of martingale-difference of $\mathcal{L}_{2}$-process indexed by $\mathcal{F}$ under the uniformly integrable entropy conditions by developing a maximal inequality as Ziegler [19] did for a triangular array of row wise independent process. The main tools are the Freedman inequality for the martingale-difference and a restricted chaining argument. Throughout the paper events are identified with their indicator functions and $E^{*}$ denotes the upper expectation with respect to the outer probability $P^{*}$.

\section{The main results}

Consider a process $\left\{S_{n}(f): f \in \mathcal{F}\right\}$ defined by

$$
S_{n}(f):=\sum_{j \leq j(n)} V_{n j}(f) \text { for } f \in \mathcal{F} .
$$

Define

$$
\sigma_{n}^{2}(f, g):=\sum_{j \leq j(n)} E_{n, j-1}\left[V_{n j}(f)-V_{n j}(g)\right]^{2} \text { for all } f, g \in \mathcal{F} .
$$

We are ready to state a maximal inequality for the martingale-difference array.

Theorem 1. Let $\left\{V_{n j}(f): j \leq j(n), n \in \mathbb{N}, f \in \mathcal{F}\right\}$ be a martingale-difference array of $\mathcal{L}_{2}$-process indexed by a class $\mathcal{F}$ of measurable functions with an envelope Fon a measurable space $(\mathbf{X}, \mathcal{X})$. Suppose that $\mathcal{F}$ has uniformly integrable entropy. Let $\mu_{n}, n \in \mathbb{N}$, be random measures on $(\mathbf{X}, \mathcal{X})$ such that

$$
P^{*}\left\{\sup _{f, g \in \mathcal{F}} \frac{\sigma_{n}^{2}(f, g)}{\left(d_{\mu_{n}}^{(2)}(f, g)\right)^{2}} \geq L\right\} \rightarrow 0, \text { as } n \rightarrow \infty \text { for a constant } L .
$$

Suppose

$$
L_{n}(\delta):=\frac{6}{\delta} \sum_{j \leq j(n)} E\left[\left(V_{n j}(F)\right)^{2}\left\{V_{n j}(F)>\delta\right\}\right] \rightarrow 0 \text { for every } \delta>0 .
$$


Then given $\epsilon>0$ and $\gamma>0$ there exists an $\eta>0$ for which

$$
\limsup _{n \rightarrow \infty} P^{*}\left(\sup _{d_{\mu_{n}}^{(2)}(f, g) \leq \eta}\left|S_{n}(f)-S_{n}(g)\right|>5 \gamma\right) \leq 3 \epsilon .
$$

Establishing a uniform CLT essentially means showing that $\mathcal{L}\left(S_{n}(f): f \in\right.$ $\mathcal{F}) \rightarrow \mathcal{L}(Z(f): f \in \mathcal{F})$, where the processes are indexed by $\mathcal{F}$ and are considered as random elements of the Banach space

$$
B(\mathcal{F}):=\left\{z: \mathcal{F} \rightarrow R:\|z\|_{\mathcal{F}}:=\sup _{f \in \mathcal{F}}|z(f)|<\infty\right\},
$$

the space of the bounded real-valued functions on $\mathcal{F}$, taken with the sup norm. The limiting process $Z=(Z(f): f \in \mathcal{F})$ is a Gaussian process whose sample paths are contained in

$$
U_{B}(\mathcal{F}, \rho):=\{z \in B(\mathcal{F}): z \text { is uniformly continuous with respect to } \rho\},
$$

where $\rho$ is a metric on $\mathcal{F}$. Notice that $\left(B(\mathcal{F}),\|\cdot\|_{\mathcal{F}}\right)$ is a Banach space and $U_{B}(\mathcal{F}, \rho)$ is a closed subspace of $\left(B(\mathcal{F}),\|\cdot\|_{\mathcal{F}}\right)$ and hence is a Banach space. In particular $U_{B}(\mathcal{F}, \rho)$ is separable if and only if $(\mathcal{F}, \rho)$ is totally bounded. Write

$$
d(f, g):=d_{\mu}^{(2)}(f, g):=\left[\int_{\mathbf{X}}(f-g)^{2} d \mu\right]^{1 / 2}
$$

where $\mu$ is a fixed measure on $(\mathbf{X}, \mathcal{X})$ with $\mu\left(F^{2}\right):=\int_{\mathbf{X}} F^{2} d \mu<\infty$. We equip the space $\mathcal{F}$ with the pseudometric $d$ so that $(\mathcal{F}, d)$ is totally bounded.

We use the following definition of weak convergence which is originally due to Hoffmann-J $\phi$ rgensen [13].

Definition 1. A sequence of $B(\mathcal{F})$-valued random functions $\left\{Y_{n}: n \geq 1\right\}$ converges in law to a $B(\mathcal{F})$-valued Borel measurable random function $Y$ whose law concentrates on a separable subset of $B(\mathcal{F})$, denoted $Y_{n} \Rightarrow Y$, if

$$
E g(Y)=\lim _{n \rightarrow \infty} E^{*} g\left(Y_{n}\right), \forall g \in C\left(B(\mathcal{F}),\|\cdot\|_{\mathcal{F}}\right),
$$

where $C\left(B(\mathcal{F}),\|\cdot\|_{\mathcal{F}}\right)$ is the set of all bounded, continuous functions from $\left(B(\mathcal{F}),\|\cdot\|_{\mathcal{F}}\right)$ into $R$.

We are ready to state the uniform CLT for a martingale difference array under the uniformly integrable entropy condition.

Theorem 2. Let $\left\{V_{n j}(f): j \leq j(n), n \in \mathbb{N}, f \in \mathcal{F}\right\}$ be an array of martingaledifference of $\mathcal{L}_{2}$-process indexed by a class $\mathcal{F}$ of measurable functions with an envelope Fon a measurable space $(\mathbf{X}, \mathcal{X})$. Suppose that $\mathcal{F}$ has uniformly integrable entropy. Assume that there exists a constant $L$ such that

$$
P^{*}\left\{\sup _{f, g \in \mathcal{F}} \frac{\sigma_{n}^{2}(f, g)}{d^{2}(f, g)} \geq L\right\} \rightarrow 0, \text { as } n \rightarrow \infty .
$$


Suppose that, as $n \rightarrow \infty$,

$$
\sum_{j \leq j(n)} v_{n j}(f) \rightarrow^{P} \sigma^{2}(f) \text { for each } f \in \mathcal{F}
$$

where $\sigma^{2}(f)$ are positive constants; and for every $\epsilon>0$,

$$
\sum_{j \leq j(n)} E_{n, j-1}\left(\left(V_{n j}(F)\right)^{2}\left\{V_{n j}(F)>\epsilon\right\}\right) \rightarrow^{P} 0 .
$$

Suppose there exists a Gaussian process $Z$ such that finite dimensional distributions of $S_{n}$ converge to those of $Z$. Then

$$
S_{n} \Rightarrow Z \text { as random elements of } B(\mathcal{F}) \text {. }
$$

The limiting process $Z=(Z(f): f \in \mathcal{F})$ is mean zero Gaussian with covariance structure $E Z(f) Z(g)$ and the sample paths of $Z$ are belong to $U_{B}(\mathcal{F}, d)$.

Proof. The result is a consequence of Theorem 1 and assumptions on the convergence of finite dimensional distributions of $S_{n}$ to those of $Z$ by applying Theorem 10. 2 of Pollard [16] to the process $\left(S_{n}(f): f \in \mathcal{F}\right)$ indexed by the totally bounded pseudometric space $(\mathcal{F}, d)$.

Remark 1. (1) A sufficient condition to the Lipsuitz condition (4) is that

$$
E^{*} \sup _{f, g \in \mathcal{F}} \frac{\sigma_{n}^{2}(f, g)}{d^{2}(f, g)} \text { converges. }
$$

(2) The condition (5) on the conditional variances and the Lindeberg condition (6) are essential in the sense that the parallel conditions are required in the one dimensional CLT.

We regain the uniform CLT for a sequence of martingale-difference in Bae and Choi [3] by applying Theorem 2 with $V_{n j}(f)=n^{-1 / 2} D_{j}(f)$.

Corollary 1 (Theorem 1 of Bae and Choi [3]). Let $\mathcal{F}$ be a class of measurable functions on a measurable space $(\mathbf{X}, \mathcal{X})$. Let $\left\{D_{j}(f): 1 \leq j \leq n, n \in \mathbb{N}, f \in \mathcal{F}\right\}$ be a sequence of martingale-difference of $\mathcal{L}_{2}$-process indexed by a class $\mathcal{F}$ with respect to an increasing sequence of $\sigma$-fields $\left\{\mathcal{E}_{j}: 0 \leq j \leq n, n \in \mathbb{N}\right\}$. Suppose that $\mathcal{F}$ has uniformly integrable entropy. Assume that there exists a constant $L$ such that

$$
P^{*}\left\{\sup _{f, g \in \mathcal{F}} \sum_{j=1}^{n} \frac{E_{j-1}\left(D_{j}(f)-D_{j}(g)\right)^{2}}{n d^{2}(f, g)} \geq L\right\} \rightarrow 0 \text { as } n \rightarrow \infty .
$$

Suppose that, as $n \rightarrow \infty$,

$$
\frac{1}{n} \sum_{j=1}^{n} E_{j-1}\left(D_{j}(f)\right)^{2} \rightarrow^{P} \sigma^{2}(f) \text { for each } f \in \mathcal{F}
$$


where $\sigma^{2}(f)$ are positive constants; and for every $\epsilon>0$

$$
\frac{1}{n} \sum_{j=1}^{n} E_{j-1}\left(\left(D_{j}(F)\right)^{2}\left\{D_{j}(F)>\epsilon \sqrt{n}\right\}\right) \rightarrow^{P} 0 .
$$

Suppose there exists a Gaussian process $Z$ such that the finite dimensional distributions of $S_{n}$ converge to those of $Z$. Then

$$
\left(\frac{1}{\sqrt{n}} \sum_{j=1}^{n} D_{j}(f): f \in \mathcal{F}\right) \Rightarrow(Z(f): f \in \mathcal{F})
$$

as random elements of $B(\mathcal{F})$. The limiting process $Z=(Z(f): f \in \mathcal{F})$ is mean zero Gaussian with covariance structure $E Z(f) Z(g)$ and the sample paths of $Z$ belong to $U_{B}(\mathcal{F}, d)$.

\section{Proof of the maximal inequality}

We will use a modified version of Freedman inequality, a maximal inequality that satisfy a Bernstein-type bound, and a restricted chaining argument. For a random variable $\xi$, we use the notation $\|\xi\|_{\infty}$ to denote the essential supremum of $|\xi|$. We also use the notation $\preceq$ to mean the left hand side is bounded by a constant times the right hand side.

Lemma 1. Let $\left(D_{j}\right)_{1 \leq j \leq n}$ be a martingale-difference with respect to increasing $\sigma$-fields $\left(\mathcal{E}_{j}\right)_{0 \leq j \leq n}$. That is, $E\left(D_{j} \mid \mathcal{E}_{j-1}\right)=0, j=1, \ldots, n$. Suppose that $\left\|D_{j}\right\|_{\infty} \leq M$ for a constant $M, j=1, \ldots, n$. Let $\tau \leq n$ be a stopping time relative to $\left(\mathcal{E}_{i}\right)$ that satisfies $\left\|\sum_{j=1}^{\tau} E\left(D_{j}^{2} \mid \mathcal{E}_{j-1}\right)\right\|_{\infty} \leq V$ for a constant $V$. If $0 \leq \eta \leq V / 2 M$, then

$$
P\left(\left|\sum_{j=1}^{\tau} D_{j}\right|>\eta\right) \leq 2 \cdot \exp \left\{-\frac{\eta^{2}}{3 V}\right\} .
$$

Proof. Since the upper bound for the tail probability $P\left(\left|\sum_{j=1}^{\tau} D_{j}\right|>\eta\right)$ in Freedman inequality, see Proposition 2.1 of Freedman [11], is given by 2 . $\exp \left[-\eta^{2} / 2(V+M \eta)\right]$ for $\eta \geq 0$, the result directly follows from the restriction $0 \leq \eta \leq V / 2 M$.

Lemma 2. Let $X_{1}, \ldots, X_{N}$ be random variables that satisfy the tail bound

$$
P\left(\left|X_{i}\right|>y\right) \leq 2 \cdot \exp \left[-\frac{1}{2} \frac{y^{2}}{V+M y}\right] \text { for every } y>0 \text { and for all } i .
$$

Then,

$$
E\left(\max _{1 \leq i \leq N}\left|X_{i}\right|\right) \preceq(M \log (1+N)+\sqrt{V} \sqrt{\log (1+N)}) .
$$

Proof. See Lemma 2.2.10 in [18]. 
We will prove the theorem by using the usual chaining argument. For this purpose we introduce some more notations. Define a covering integral

$$
J(\delta)=\int_{0}^{\delta}\left[2 \ln \frac{N^{2}(\epsilon)}{\epsilon}\right]^{1 / 2} d \epsilon .
$$

Notice that the uniformly integrable entropy condition implies the finiteness of covering integral $J(\cdot)$.

Lemma 3. Suppose the metric space $(\mathcal{F}, d)$ has a finite covering integral $J(\cdot)$. Let $\{Z(f): f \in \mathcal{F}\}$ be a stochastic process that satisfies the exponential inequality, for a constant $D$,

$$
P\{|Z(f)-Z(g)|>\eta\} \leq 2 \cdot \exp \left\{-\frac{\eta^{2}}{2 D^{2} \delta^{2}}\right\} \text { if } d(f, g) \leq \delta
$$

for every $\eta>0$ and $\delta>0$ with $\delta \geq \alpha \eta^{2}$, for some constant $\alpha$. Let $\mathcal{F}(\alpha)$ be a $\alpha$-net(containing $N(\alpha)$ points) for $\mathcal{F}$; let $f_{\alpha}$ be the closest point in $\mathcal{F}(\alpha)$ to $f$. Then given $\epsilon>0$ and $\gamma>0$, there exists $\delta>0$, depending on $\epsilon, \gamma$, and $J(\cdot)$, for which

$$
P\left\{\sup _{d(f, g)<\delta}|Z(f)-Z(g)|>5 \gamma\right\} \leq 2 \epsilon+P\left\{\sup _{\mathcal{F}}\left|Z(f)-Z\left(f_{\alpha}\right)\right|>\gamma\right\}
$$

provided $\alpha \leq \frac{1}{3} \epsilon$ and $\gamma \leq 144$ and $J(\alpha) \leq \min \{\gamma / 12 D, 3 / D\}$.

Proof. See Theorem 26, p. 169 in [15].

We introduce the following truncation argument. For $\delta>0$, let

$$
f^{(\delta)}(\cdot)= \begin{cases}\delta & \text { if } f(\cdot)>\delta \\ f(\cdot) & \text { if }|f(\cdot)| \leq \delta \\ -\delta & \text { if } f(\cdot)<-\delta\end{cases}
$$

so that $f^{(\delta)}(\cdot)$ is a truncation of $f(\cdot)$ at the level $\delta$. For fixed $\delta>0$, for $j \leq$ $j(n)$, and $n \in \mathbb{N}$, we simplify the notation by writing $\overline{V_{n j}\left(f^{(\delta)}\right)}:=V_{n j}\left(f^{(\delta)}\right)-$ $E_{n, j-1} V_{n j}\left(f^{(\delta)}\right)$, and define

$$
S_{n}^{(\delta)}(f)=\sum_{j \leq j(n)} \overline{V_{n j}\left(f^{(\delta)}\right)} \text { for } f \in \mathcal{F} .
$$

Proposition 1. Let $\left\{V_{n j}(f): j \leq j(n), n \in \mathbb{N}, f \in \mathcal{F}\right\}$ be a martingaledifference array of $\mathcal{L}_{2}$-process indexed by a class $\mathcal{F}$ of measurable functions with an envelope $F$ on a measurable space $(\mathbf{X}, \mathcal{X})$. Suppose that $\mathcal{F}$ has uniformly integrable entropy. Let $\mu_{n}, n \in \mathbb{N}$, be random measures on $(\mathbf{X}, \mathcal{X})$ and let $\tau_{n} \leq n$ be a sequence of stopping times relative to the $\sigma$-fields $\left\{\mathcal{E}_{n j}: 0 \leq j \leq\right.$ $j(n), n \in \mathbb{N}\}$ that satisfies almost surely

$$
\sigma_{\tau_{n}}^{2}(f, g) \leq L\left(d_{\mu_{n}}^{(2)}(f, g)\right)^{2} \text { for } f, g \in \mathcal{F} \text { and for a constant } L .
$$


Then given $\epsilon>0$ and $\gamma>0$ there exists a $\eta>0$ such that for every $n \in \mathbb{N}$,

$$
P^{*}\left\{\sup _{d_{\mu_{n}}^{(2)}(f, g) \leq \eta}\left|S_{\tau_{n}}^{(\delta)}(f)-S_{\tau_{n}}^{(\delta)}(g)\right|>5 \gamma\right\}<3 \epsilon+P\left(\tau_{n} \neq n\right) .
$$

Proof. Let $\delta>0$. Let $f, g \in \mathcal{F}$ be fixed. Then the martingale-difference $D_{j}:=\overline{V_{n j}\left(f^{(\delta)}\right)}-\overline{V_{n j}\left(g^{(\delta)}\right)}$ have an upper bound $M:=4 \delta$. On the almost sure event $\left\{\sigma_{\tau_{n}}^{2}(f, g) \leq L\left(d_{\mu_{n}}^{(2)}(f, g)\right)^{2}\right\}$, taking $V:=5 L\left(d_{\mu_{n}}^{(2)}(f, g)\right)^{2}$, we observe that

$$
\begin{aligned}
\sum_{j \leq \tau_{n}} E_{n, j-1}\left[\overline{V_{n j}\left(f^{(\delta)}\right)}-\overline{V_{n j}\left(g^{(\delta)}\right)}\right]^{2} & \leq \sum_{j \leq \tau_{n}} E_{n, j-1}\left[V_{n j}(f)-V_{n j}(g)\right]^{2} \\
& =\sigma_{\tau_{n}}^{2}(f, g) \leq L\left(d_{\mu_{n}}^{(2)}(f, g)\right)^{2} \leq V .
\end{aligned}
$$

Write $2 D^{2}=15 L$ and take $\alpha^{2}=\frac{60 \delta}{D^{2}}$. By Lemma 1 we have that, if $\delta \geq \alpha \eta^{1 / 2}$ then

$$
P\left(\left|S_{\tau_{n}}^{(\delta)}(f)-S_{\tau_{n}}^{(\delta)}(g)\right|>\eta\right) \leq 2 \exp \left\{-\frac{\eta^{2}}{2 D^{2} \delta^{2}}\right\} \text { for } d_{\mu_{n}}^{(2)}(f, g) \leq \delta .
$$

Now let $\epsilon>0$ and $\gamma>0$. Then, by Lemma 3, there exists a $\delta>0$ for which

$$
\begin{aligned}
& P\left\{\sup _{d_{\mu_{n}}^{(2)}(f, g) \leq \delta}\left|S_{\tau_{n}}^{(\delta)}(f)-S_{\tau_{n}}^{(\delta)}(g)\right|>5 \gamma\right\} \\
\leq & 2 \epsilon+P\left\{\sup _{\mathcal{F}}\left|S_{\tau_{n}}^{(\delta)}(f)-S_{\tau_{n}}^{(\delta)}\left(f_{\alpha}\right)\right|>\gamma\right\},
\end{aligned}
$$

provided $\alpha \leq \frac{1}{3} \epsilon$ and $\gamma \leq 144$ and $J(\alpha) \leq \min \{\gamma / 12 D, 3 / D\}$. Write $\mathcal{H}:=$ $\left\{f-f_{\alpha}: f \in \mathcal{F}\right\}$. We assume without loss of generality that $E_{n, j-1} h=0$ for every $h \in \mathcal{H}$. Otherwise subtract and add the term $E_{n, j-1} h$ as above.

Claim 1. $P\left\{\sup _{h \in \mathcal{H}}\left|S_{\tau_{n}}^{(\delta)}(h)\right|>\gamma\right\}<\epsilon$.

Introduce a random semimetric $d_{n}$ defined as

$$
d_{n}^{2}(g, h):=\sum_{j \leq j(n)}\left(V_{n j}(g)-V_{n j}(h)\right)^{2} \text { for } g, h \in \mathcal{F} .
$$

Given $\alpha>0$, find functions $g_{1}, \ldots, g_{M}$ in $\mathcal{H} \subseteq H_{0} \ominus H_{-1}$ (see Bae [2]), where $M:=N(\alpha):=N\left(\alpha, \mathcal{H}, d_{n}\right)$ for which

$$
\min _{1 \leq i \leq M}\left[d_{n}^{2}\left(h-g_{i}\right)\right]^{1 / 2} \leq \alpha \text { for every } h \in \mathcal{H} .
$$

Recall

$$
S_{n}^{(\delta)}(h):=\sum_{j \leq j(n)} V_{n j}(h)
$$


For each $g$ and $h,\left|S_{n}^{(\delta)}(h)\right| \leq\left[d_{n}^{2}(h, g)\right]^{1 / 2}+\left|S_{n}^{(\delta)}(g)\right|$. For $h \in \mathcal{H}$ set $g$ equal to the $g_{i}$ that minimizes $\left[d_{n}\left(h-g_{i}\right)^{2}\right]^{1 / 2}$. Then

$$
\left|S_{n}^{(\delta)}(h)\right| \leq \alpha+\left|S_{n}^{(\delta)}\left(g_{i}\right)\right|
$$

whence

$$
\sup _{h \in \mathcal{H}}\left|S_{n}^{(\delta)}(h)\right| \leq \alpha+\max _{1 \leq i \leq M}\left|S_{n}^{(\delta)}\left(g_{i}\right)\right|
$$

Therefore

$$
P\left\{\sup _{h \in \mathcal{H}}\left|S_{n}^{(\delta)}(h)\right|>2 \alpha\right\} \leq P\left\{\max _{1 \leq i \leq M}\left|S_{n}^{(\delta)}\left(g_{i}\right)\right|>\alpha\right\} .
$$

Now, applying (7) for the second inequality, we see that

$$
\begin{aligned}
P\left\{\sup _{h \in \mathcal{H}}\left|S_{\tau_{n}}^{(\delta)}(h)\right|>2 \alpha\right\} \leq & P\left\{\max _{1 \leq i \leq M}\left|S_{\tau_{n}}^{(\delta)}\left(g_{i}\right)\right|>\alpha\right\} \\
& +P\left\{\tau_{n} \neq n\right\} .
\end{aligned}
$$

For each $i=1, \ldots, N(\alpha),\left\|g_{i}\right\|_{\infty} \leq 4 \delta$ and $\left\|\sum_{j=1}^{\tau_{n}} E_{j-1} g_{i}^{2}\right\|_{\infty} \leq 15 L \delta^{2}$. Applying Freedman inequality, Proposition 2.1 of Freedman [11],

$$
P\left(\left|S_{\tau_{n}}^{(\delta)}\left(g_{i}\right)\right|>y\right) \leq 2 \exp \left[-\frac{1}{2} \frac{y^{2}}{15 L \delta^{2}+4 \delta y}\right]
$$

for every $y>0$. Therefore applying Markov inequality followed by Lemma 2 we have that

$$
\begin{aligned}
& P\left\{\sup _{h \in \mathcal{H}}\left|S_{\tau_{n}}^{(\delta)}(h)\right|>2 \gamma\right\}-P\left\{\tau_{n} \neq n\right\} \\
\leq & \frac{1}{\gamma} E\left\{\max _{1 \leq i \leq N(\alpha)}\left|S_{\tau_{n}}^{(\delta)}\left(g_{i}\right)\right|\right\} \\
\preceq & \left(4 \delta \log (1+N(\alpha))+\sqrt{15 L \delta^{2}} \sqrt{\log (1+N(\alpha))}\right) \\
\preceq & \int_{0}^{\delta}\left[\ln N\left(\epsilon, \mathcal{F}, d_{n}\right)\right]^{1 / 2} d \epsilon \\
\preceq & \int_{0}^{C \delta} \sup _{\gamma \in M(\mathbf{X}, F)}\left[\ln N\left(\epsilon\left[\gamma\left(F^{2}\right)\right]^{1 / 2}, \mathcal{F}, d_{\gamma}^{(2)}\right)\right]^{1 / 2} d \epsilon
\end{aligned}
$$

for a constant $C$. Now due to the uniformly integrable entropy condition we can choose $\delta>0$ so that the last integral is less than $\epsilon$. The proof of Claim 1 is completed. Hence the proof of Proposition 1 is completed.

We are now ready to finish the proof of Theorem 1.

Proof. Since $\left\{V_{n j}(f), \mathcal{E}_{n j}\right\}$ is a martingale-difference, for any $\delta>0$, using the identity

$$
\left|E_{n, j-1}\left(V_{n j}(f)\left\{\left|V_{n j}(f)\right|>\delta\right\}\right)\right|=\left|E_{n, j-1}\left(V_{n j}(f)\left\{\left|V_{n j}(f)\right| \leq \delta\right\}\right)\right|,
$$


we have

$$
\begin{aligned}
\sup _{f \in \mathcal{F}}\left|S_{n}(f)-S_{n}^{(\delta)}(f)\right| \leq & \frac{1}{\delta} \sum_{j \leq j(n)}\left(V_{n j}(f)\right)^{2}\left\{V_{n j}(f)>\delta\right\} \\
& +\frac{2}{\delta} \sum_{j \leq j(n)} E_{n, j-1}\left(\left(V_{n j}(f)\right)^{2}\left\{V_{n j}(f)>\delta\right\}\right) .
\end{aligned}
$$

For any $\delta>0$, and $0<\eta \leq\left(\frac{L \delta^{2}}{12}\right)^{1 / 2}$, we get

$$
\begin{aligned}
& \sup _{\sigma_{n}(f, g) \leq \eta}\left|S_{n}(f)-S_{n}(g)\right| \\
\leq & \sup _{\sigma_{n}(f, g) \leq \eta}\left|S_{n}^{(\delta)}(f)-S_{n}^{(\delta)}(g)\right|+2 \sup _{f \in \mathcal{F}}\left|S_{n}(f)-S_{n}^{(\delta)}(f)\right| \\
\leq & \sup _{\sigma_{n}(f, g) \leq \eta}\left|S_{n}^{(\delta)}(f)-S_{n}^{(\delta)}(g)\right|+\frac{2}{\delta} \sum_{j \leq j(n)}\left(V_{n j}(f)\right)^{2}\left\{V_{n j}(f)>\delta\right\} \\
& +\frac{4}{\delta} \sum_{j \leq j(n)} E_{n, j-1}\left(V_{n j}(f)\right)^{2}\left\{V_{n j}(f)>\delta\right\} .
\end{aligned}
$$

Therefore we have

$$
E^{*} \sup _{\sigma_{n}(f, g) \leq \eta}\left|S_{n}(f)-S_{n}(g)\right| \leq E^{*} \sup _{\sigma_{n}(f, g) \leq \eta}\left|S_{n}^{(\delta)}(f)-S_{n}^{(\delta)}(g)\right|+L_{n}(\delta) .
$$

Define a stopping time $\tau_{n}$ by, for $n \geq 1$

$$
\tau_{n}:=n \wedge \max \left\{k \geq 0: \sup _{f, g \in \mathcal{F}} \frac{\sigma_{k}^{2}(f, g)}{\left(d_{\mu_{n}}^{(2)}(f, g)\right)^{2}}<L\right\} .
$$

Being the random variables $\sigma_{k}^{2}(f, g)$ predictable, we see that $\tau_{n}$ is a stopping time. Notice that $P^{*}\left(\tau_{n}<n\right) \rightarrow 0$ as $n \rightarrow \infty$. Therefore, it is enough to prove our theorem for the stopped process $\left\{S_{\tau_{n}}\right\}$.

Claim 2. Given $\epsilon>0$ and $\gamma>0$ there exists a $\eta>0$ such that

$$
\limsup _{n \rightarrow \infty} P^{*}\left\{\sup _{\substack{(2) \\ d_{\mu_{n}}(f, g) \leq \eta}}\left|S_{\tau_{n}}^{(\delta)}(f)-S_{\tau_{n}}^{(\delta)}(g)\right|>5 \gamma\right\}<3 \epsilon .
$$

The Claim follows from Proposition 1. The proof of Theorem 1 is completed.

\section{A sequential empirical process for martingale-difference}

In this section, we consider a uniform CLT for classes of functions changing with $n$. See the Section 2.11.3 of Van der Vaart and Wellner [18]. Let $x \mapsto$ $f_{n, t}(x)$ be functions from $\mathcal{X}$ to $R$ indexed by $n \in \mathbb{N}$ and a fixed, totally bounded semimetric space $(T, \rho)$.

Consider for each $n \in \mathbb{N}$, let $\mathcal{F}_{n}=\left\{f_{n, t}: t \in T\right\} \subseteq H_{0} \ominus H_{-1}$ with envelope functions $F_{n}$. Write as before $\xi_{j}:=T^{j}(X), V_{0}:=T^{0}(X)(=X)$. From our setup 
it follows that for each $n \in \mathbb{N}$ and $t \in T,\left\{f_{n, t}\left(\xi_{j}\right), M_{j}\right\}$ forms a martingaledifference arrays. Consider the stochastic process $\left\{Z_{n} f_{n, t}: t \in T\right\}$ defined by

$$
Z_{n}\left(f_{n, t}\right)=\frac{1}{\sqrt{n}} \sum_{j=1}^{n} f_{n, t}\left(\xi_{j}\right) \text { for } t \in T .
$$

Therefore this situation fits the general set-up of martingale-difference arrays by setting

$$
V_{n, j}(t)=\frac{1}{\sqrt{n}} f_{n, t}\left(\xi_{j}\right)
$$

and

$$
\mathcal{E}_{n, j}=\mathcal{M}_{j} .
$$

Given envelope functions $F_{n}$, assume that $P^{*} F_{n}^{2}=O(1)$ and $P^{*} F_{n}^{2}\left\{F_{n}>\right.$ $\eta \sqrt{n}\} \rightarrow 0$ for every $\eta>0$. Then we get the following central limit theorem.

Theorem 3. For each $n \in \mathbb{N}$, let $\mathcal{F}_{n}=\left\{f_{n, t}: t \in T\right\} \subseteq H_{0} \ominus H_{-1}$ be a class of measurable functions indexed by a totally bounded semimetric space $(T, \rho)$. Suppose that

$$
\sup _{P} \int_{0}^{\delta_{n}}\left[\ln N\left(\epsilon\left\|F_{n}\right\|_{P, 2}, \mathcal{F}_{n}, L_{2}(P)\right)\right]^{1 / 2} d \epsilon \rightarrow 0 \text { for every } \delta_{n} \downarrow 0 .
$$

Suppose there exists a constant $L>0$ such that

$$
P^{*}\left(\sup _{s, t \in T} \sum_{j=1}^{n} \frac{E_{j-1}\left[f_{n, s}\left(\xi_{j}\right)-f_{n, t}\left(\xi_{j}\right)\right]^{2}}{n \rho^{2}(s, t)} \geq L\right) \rightarrow 0 .
$$

Then $\left\{Z_{n} f_{n, t}: t \in T\right\}$ converges in distribution to a tight Gaussian process as random elements of $B(T)$ provided the sequence of covariance functions $E f_{n, s} f_{n, t}-E f_{n, s} E f_{n, t}$ converges pointwise on $T \times T$

Remark 2. In order to see that our result generalizes that of IID problem, see Theorem 2.11.22 of Van der Vaart and Wellner [18], let $\xi$ be a random variable on a probability space $\left(S, \mathcal{B}, P_{0}\right)$, and let $\left\{\xi_{i}, i \geq 1\right\}$ be a sequence of independent copies of $\xi$. Suppose that $E_{0} f_{n, t}(\xi)=0$ for all $t \in T$. Consider $P=\left(P_{0}\right)^{Z}$ so that $\left(X_{i}\right)$ are IID. In this case we see that

$$
E_{j-1}\left[f_{n, s}\left(\xi_{j}\right)-f_{n, t}\left(\xi_{j}\right)\right]^{2}=E_{0}\left[f_{n, t}(\xi)-f_{n, t}(\xi)\right]^{2} .
$$

So the condition (10) is equivalent to the condition

$$
\sup _{\rho(s, t)<\delta_{n}} P\left(f_{n, s}-f_{n t}\right)^{2} \rightarrow 0 \text { for every } \delta_{n} \downarrow 0 .
$$

Therefore, Theorem 2.11.22 of Van der Vaart and Wellner [18] will be a special case of Theorem 3 . 


\section{References}

[1] R. J. Adler, An introduction to continuity, extrema, and related topics for general Gaussian processes, Institute of Mathematical Statistics Lecture Notes-Monograph Series, 12. Institute of Mathematical Statistics, Hayward, CA, 1990.

[2] J. Bae, An empirical CLT for stationary martingale differences, J. Korean Math. Soc. 32 (1995), no. 3, 427-446.

[3] J. Bae and M. J. Choi, The uniform CLT for martingale difference of function-indexed process under uniformly integrable entropy, Commun. Korean Math. Soc. 14 (1999), no. $3,581-595$.

[4] J. Bae and S. Levental, Uniform CLT for Markov chains and its invariance principle: a martingale approach, J. Theoret. Probab. 8 (1995), no. 3, 549-570.

[5] R. F. Bass, Law of the iterated logarithm for set-indexed partial sum processes with finite variance, Z. Wahrsch. Verw. Gebiete 70 (1985), no. 4, 591-608.

[6] M. D. Donsker, An invariance principle for certain probability limit theorems, Mem. Amer. Math. Soc. 1951 (1951), no. 6, 12 pp.

[7] P. Doukhan, P. Massart, and E. Rio, Invariance principles for absolutely regular empirical processes, Ann. Inst. H. Poincare Probab. Statist. 31 (1995), no. 2, 393-427.

[8] R. M. Dudley, Donsker classes of functions, Statistics and related topics (Ottawa, Ont., 1980), pp. 341-352, North-Holland, Amsterdam-New York, 1981

[9] _ Uniform Central Limit Theorems, Cambridge Studies in Advanced Mathematics, 63. Cambridge University Press, Cambridge, 1999

[10] R. Durrett, Probability: Theory and Examples, Wadsworth \& Brooks/Cole Advanced Books \& Software, Pacific Grove, CA, 1991.

[11] D. Freedman, On tail probabilities for martingales, Ann. Probability 3 (1975), 100-118.

[12] M. I. Gordin and B. A. Lifsic, The central limit theorem for stationary Markov Processes, Soviet Math. Dokl. 19 (1978), no. 2, 392-394.

[13] J. Hoffmann-Jørgensen, Stochastic Processes on Polish Spaces, Various Publications Series (Aarhus), 39. Aarhus Universitet, Matematisk Institut, Aarhus, 1991.

[14] M. Ossiander, A central limit theorem under metric entropy with $L_{2}$ bracketing, Ann. Probab. 15 (1987), no. 3, 897-919.

[15] D. Pollard, Convergence of Stochastic Processes, Springer-Verlag, New York, 1984.

[16] _ Empirical Processes: Theory and Applications, NSF-CBMS Regional Conference Series in Probability and Statistics, 2. Institute of Mathematical Statistics, Hayward, CA; American Statistical Association, Alexandria, VA, 1990.

[17] S. van der Geer, Empirical Processes in M-Estimation, Cambridge University Press, 2000.

[18] A. W. van der Vaart and J. A. Wellner, Weak Convergence and Empirical Processes, Springer Series in Statistics. Springer-Verlag, New York, 1996.

[19] K. Ziegler, Functional central limit theorems for triangular arrays of function-indexed processes under uniformly integrable entropy conditions, J. Multivariate Anal. 62 (1997), no. $2,233-272$

JONGSIG BAE

Department of Mathematics and Institute of Basic Science

SUNGKYUNKWAN UNIVERSITY

SUWON 440-746, KorEA

E-mail address: jsbae@skku.edu 


\section{DOOBAE Jun}

Department of MATHEMATics

SUNGKYUNKWAN UNIVERSITY

SuWON 440-746, KorEA

E-mail address: dbjun@skku.edu

Shlomo LeVEntal

Department of Statistics and Probability

Michigan State University

EAST LANSing, Michigan 48824, U.S.A.

E-mail address: levental@stt.msu.edu 